\title{
A remark on soliton equation of mean curvature flow
}

\author{
LI MA and YANG YANG \\ Department of Mathematics, Tsinghua University, 100084 Beijing, China \\ Manuscript received on February 4, 2004; accepted for publication on February 8, 2004; \\ presented by MANFREDO DO CARMO
}

\begin{abstract}
In this note, we consider self-similar immersions of the mean curvature flow and show that a graph solution of the soliton equation, provided it has bounded derivative, converges smoothly to a function which has some special properties (see Theorem 1.1).
\end{abstract}

Key words: soliton, Self-Similar, Mean curvature flow.

\section{INTRODUCTION}

Let $M^{n+k}$ be a Riemannian manifold of dimension $n+k$. Assume that $\Sigma^{n}$ be a Riemannian manifold of dimension $n$ without boundary. Let $F: \Sigma^{n} \rightarrow M^{n+k}$ be an isometric immersion. Denote $\nabla$ (respectively $D$ ) the covariant differentiation on $\Sigma$ (on $M$ ). Let $T \Sigma$ and $N \Sigma$ be the tangent bundle and normal bundle of $\Sigma$ in $M$ respectively. We define the second fundamental form of the immersion $\Sigma$ by

$$
I I: T \Sigma \times T \Sigma \rightarrow N \Sigma
$$

with

$$
I I(X, Y)=D_{X} Y-\nabla_{X} Y
$$

for tangential vector fields $X, Y$ on $\Sigma$. We define the mean curvature vector field (in short, MCV) by

$$
\bar{H}=\operatorname{tr}_{\Sigma} I I
$$

In recent years, many people are interested in studying the evolution of the immersion $F$ : $\Sigma^{n} \rightarrow M^{n+k}$ along its Mean Curvature Flow (in short, just say MCF). The MCF is defined as 
follows. Given an one-parameter family of sub-manifolds $\Sigma_{t}=F_{t}(\Sigma)$ with immersions $F_{t}$ : $\Sigma \longrightarrow M$. Let $\bar{H}(t)$ be the MCV of $\Sigma_{t}$. Then our MCF is the equation/system

$$
\frac{\partial F(x, t)}{\partial t}=\bar{H}(x, t) .
$$

This flow has many very nice results if the codimension $k=1$. See the work of Huisken 1993 for a survey in this regard. Since there is very few result about MCF in higher codimension, we will study it in the target when $M^{n+k}=R^{n+k}$, which is the standard Euclidian space.

In this short note, we will consider a family of self-similar graphic immersions $F(\cdot, t): \mathbb{R}^{n} \rightarrow$ $\mathbb{R}^{n+k}$ of the Mean Curvature Flow (MCF):

$$
\frac{\partial}{\partial t} F(x, t)=\bar{H}(x, t), \quad \forall x \in \mathbb{R}^{n}, \quad \forall t \in(-\infty, 0) .
$$

Write

$$
\Sigma_{t}=F\left(\mathbb{R}^{n}, t\right)
$$

and

$$
F=\left(F^{A}\right), \quad 1 \leq A \leq n+k .
$$

By definition, we call the family $\Sigma_{t}$ self-similar if

$$
\Sigma_{t}=\sqrt{-t} \Sigma_{-1}, \quad \forall t<0
$$

In this case, we can reduce the MCF into an elliptic system. In the other word, we have the following parametric elliptic equation for the family $\Sigma_{t}$ :

$$
\bar{H}(x)+F^{\perp}(x)=0, \quad \forall x \in \Sigma_{-1}:=\Sigma .
$$

We will call this system as the soliton equation of the MCF. Note that this equation is usually obtained from the monotonicity formula of Huisken 1989 for blow-up. It is a hard and open problem to classify solutions of this equation.

Fix $\Sigma=\Sigma_{t}$. Assume that $F(x)=(x, f(x))$. Let

$$
Q=\left(Q_{\alpha}^{A}\right), \quad n+1 \leq \alpha \leq n+k \quad 1 \leq A \leq n+k
$$

is the orthogonal projection onto $N_{p} \Sigma$, where $p \in \Sigma$. Then the second fundamental form of $\Sigma$ can be written as

$$
\mathrm{II}_{i j}^{A}=Q_{\alpha}^{A} D_{i j}^{2} f^{\alpha}
$$

Hence, we have the expression for the mean curvature vector of $\Sigma$ in $\mathbb{R}^{n+k}$ :

$$
\bar{H}^{A}=g^{i j} Q_{\alpha}^{A} D_{i j}^{2} f^{\alpha}
$$

Our main result in this paper is the following 
THEOREM 1.1. Let $F(x)=(x, f(x)), x \in \mathbb{R}^{n}$ be a graph solution to the soliton equation

$$
\bar{H}(x)+F^{\perp}(x)=0 .
$$

Assume $\sup _{\mathbb{R}^{n}}|D f(x)| \leq C_{0}<+\infty$. Then there exists a unique smooth function $f_{\infty}: \mathbb{R}^{n} \rightarrow \mathbb{R}^{k}$ such that

$$
f_{\infty}(x)=\lim _{\lambda \rightarrow \infty} f_{\lambda}(x)
$$

and

$$
f_{\infty}(r x)=r f_{\infty}(x)
$$

for any real number $r \neq 0$, where

$$
f_{\lambda}(x)=\lambda^{-1} f(\lambda x)
$$

We remark that the proof of this result given below is very simple. But it is based on a nice observation. We just use the divergence theorem with a nice test function. In the next section, we recall the form of divergence theorem for convenient of the readers. In the last section we give a proof of our Theorem.

We point out that we may consider $F_{\infty}(x)=\left(x, f_{\infty}(x)\right)$ obtained above as a tangential minimal cone along the research direction done by Simon 1983 (see also Ecker and Huisken 1989).

\section{PRELIMINARY}

Given a vector field $X: \Sigma \rightarrow T M$. Let $X^{T}$ and $X^{N}$ denote the projection of $X$ onto $T \Sigma$ and $N \Sigma$ respectively. We define the divergence of $X$ on $\Sigma$ as

$$
\operatorname{div}_{\Sigma} X=\sum g^{i j}\left\langle D_{i} X, \frac{\partial}{\partial x^{j}}\right\rangle
$$

where $\left(g^{i j}\right)=\left(g_{i j}\right)^{-1}$, and $\left(g_{i j}\right)$ is the induced metric tensor written in local coordinates $\left(x^{i}\right)$ on $\Sigma$.

Note that, for any tangential vector field $Y$ on $\Sigma$,

$$
D_{Y} X=D_{Y} X^{T}+D_{Y} X^{N}
$$

So

$$
\begin{aligned}
\left\langle D_{Y} X, Y\right\rangle & =\left\langle D_{Y} X^{T}, Y\right\rangle+\left\langle D_{Y} X^{N}, Y\right\rangle \\
& =\left\langle\nabla_{Y} X^{T}, Y\right\rangle-\left\langle D_{Y} Y, X^{N}\right\rangle \\
& =\left\langle\nabla_{Y} X^{T}, Y\right\rangle-\langle I I(Y, Y), X\rangle .
\end{aligned}
$$

Hence

$$
\operatorname{div}_{\Sigma} X^{T}=\operatorname{div}_{\Sigma} X+\langle X, \bar{H}\rangle,
$$


and by the Stokes formula on $\Sigma$, we have

$$
\int_{\Sigma} \operatorname{div} X^{T}=\int_{\partial_{\Sigma}}\langle X, v\rangle d \sigma
$$

and

$$
\int_{\Sigma} \operatorname{div}_{\Sigma} X d v=-\int_{\Sigma}\langle\bar{H}, X\rangle d v+\int_{\partial \Sigma}\langle X, v\rangle d \sigma
$$

where $v$ is the exterior normal vector field to $\Sigma$ on $\partial \Sigma$.

\section{PROOF OF MAIN THEOREM}

In the following, we take $M^{n+k}=\mathbb{R}^{n+k}$ as the standard Euclidean space. We assume that the assumption of our Theorem 1.1 is true in this section.

Define the vector field

$$
X=-(1+|F|)^{-s} F
$$

where $s \in \mathbb{R}$ to be determined.

Note that, $\nabla|F|=\frac{F^{\top}}{|F|}$ and $\operatorname{div}_{\Sigma} F=n$. So

$$
\begin{aligned}
\operatorname{div}_{\Sigma} X & =-\left\langle\nabla(1+|F|)^{-s}, F\right\rangle-(1+|F|)^{-s} \operatorname{div}_{\Sigma} F \\
& =\frac{s(1+|F|)^{-s-1}}{|F|}\left|F^{\top}\right|^{2}-n(1+|F|)^{-s}
\end{aligned}
$$

Locally, we may assume that $\Sigma$ is a graph of the form $(x, f(x)) \in B_{R}(0) \times \mathbb{R}^{k}$, where $B_{R}(0)$ is the ball of radius $R$ centered at 0 . Let $\Sigma_{R}=\Sigma \cap\left(B_{R}(0) \times \mathbb{R}^{k}\right)$. By the divergence theorem we have $(d)$ :

$$
\int_{\Sigma_{R}} \operatorname{div}_{\Sigma} X=-\int_{\Sigma_{R}}\langle\bar{H}, X\rangle+\int_{\partial \Sigma_{R}}\langle X, v\rangle
$$

Clearly we have that the left side of $(d)$ is

$$
\int_{\Sigma_{R}} \operatorname{div}_{\Sigma} X=s \int_{\Sigma_{R}} \frac{(1+|F|)^{-s-1}}{|F|}\left|F^{\top}\right|^{2}-n \int_{\Sigma_{R}}(1+|F|)^{-s}
$$

By direct computation, the right side of $(d)$ is

$$
\begin{aligned}
-\int_{\Sigma_{R}}\langle\bar{H}, X\rangle+\int_{\partial \Sigma_{R}}\langle X, v\rangle & =\int_{\Sigma_{R}}(1+|F|)^{-s}\left|F^{\perp}\right|^{2}+\int_{\partial_{\Sigma_{R}}}(1+|F|)^{-s}\langle F, v\rangle \\
& =\int_{\Sigma_{R}}(1+|F|)^{-s}|\bar{H}|^{2}+\int_{\partial_{\Sigma_{R}}}(1+|F|)^{-s}\langle F, v\rangle .
\end{aligned}
$$

Hence, we have

$$
\int_{\Sigma_{R}}(1+|F|)^{-s}|\bar{H}|^{2}=s \int_{\Sigma_{R}} \frac{(1+|F|)^{-s-1}}{|F|}\left|F^{\top}\right|^{2}-n \int_{\Sigma_{R}}(1+|F|)^{-s}-\int_{\partial_{\Sigma_{R}}}(1+|F|)^{-s}\langle F, v\rangle .
$$


Since $\left|F^{\top}\right| \leq|F| \leq 1+|F|$, we have

$$
\int_{\Sigma_{R}} \frac{(1+|F|)^{-s-1}}{|F|}\left|F^{\top}\right|^{2} \leq \int_{\Sigma_{R}}(1+|F|)^{-s} .
$$

Clearly we have

$$
\left|\int_{\partial_{\Sigma_{R}}}(1+|F|)^{-s}\langle F, v\rangle\right| \leq \int_{\partial_{\Sigma_{R}}}(1+|F|)^{1-s} .
$$

Combining these two inequalities together we get

$$
\int_{\Sigma_{R}}(1+|F|)^{-s}|\bar{H}|^{2} \leq(s-n) \int_{\Sigma_{R}}(1+|F|)^{-s}+\int_{\partial_{\Sigma_{R}}}(1+|F|)^{1-s} .
$$

Choosing $s=n$ yields $(*)$ :

$$
\int_{\Sigma_{R}}(1+|F|)^{-n}|\bar{H}|^{2} \leq \int_{\partial_{\Sigma_{R}}}(1+|F|)^{1-n} .
$$

By our assumption we have that $\exists C>0$ such that for $F(x)=(x, f(x))$ on $\Sigma=\mathbb{R}^{n}$, we have

$$
\operatorname{det}\left(I+(d f)^{\top} d f\right) \leq C
$$

on $\Sigma$. Since

$$
g_{i j}=\delta_{i j}+D_{i} f^{\alpha} \cdot D_{j} f^{\alpha},
$$

we know that

$$
I \leq\left(g_{i j}\right) \leq C I
$$

Hence

$$
(1+|x|) \leq(1+|F(x)|) \leq C(1+|x|) .
$$

Therefore we get from $(*)$ the key estimate $(K)$ :

$$
\int_{B_{R}(0)}(1+|x|)^{-n}|\bar{H}|^{2} d x \leq C \int_{\partial B_{R}(0)}(1+|x|)^{1-n} \leq C .
$$

We now go to the proof of our Theorem 1.1.

Proof. Note that the mean curvature flow for the graph of $f$ can be read as

$$
\frac{\partial f^{\alpha}}{\partial t}=g^{i j} D_{i j}^{2} f^{\alpha}, \alpha=1, \cdots, k
$$

The important fact about this equation is that it is invariant under the transformation

$$
f(x) \rightarrow \frac{1}{\lambda} f(\lambda x), \forall \lambda>0
$$


Compute

$$
\begin{aligned}
\frac{d}{d \lambda} f_{\lambda}(x) & =-\lambda^{-2} f(\lambda x)+\lambda^{-1} D f(\lambda x) \cdot x \\
& =\lambda^{-2}[D f(\lambda x) \cdot \lambda x-f(\lambda x)] \\
& =\lambda^{-2}\langle(D f(\lambda x),-1),(\lambda x, f(\lambda x))\rangle \\
& =\lambda^{-2}\langle(D f(\lambda x),-1), F(\lambda x)\rangle \\
& =\lambda^{-2}\left\langle(D f(\lambda x),-1), F(\lambda x)^{\perp}\right\rangle .
\end{aligned}
$$

Here we have used the fact that

$$
(D f(\lambda x),-1) \perp T_{p} \Sigma .
$$

So

$$
\frac{d}{d \lambda} f_{\lambda}(x)=\lambda^{-2}\langle(-D f(\lambda x), 1), \bar{H}\rangle
$$

Hence

$$
\left|\frac{d}{d \lambda} f_{\lambda}(x)\right| \leq C \lambda^{-2}|\bar{H}|
$$

So, for $x \in S^{n-1}$, we have

$$
\begin{aligned}
\left|f_{\lambda}(x)-f_{\mu}(x)\right| & \leq C \int_{\lambda}^{\mu} \frac{\bar{H}(\lambda x)}{\sigma^{2}} d \sigma \\
& \leq C\left(\int_{\lambda}^{\mu} \frac{1}{\sigma^{3}} d \sigma\right)\left(\int_{\lambda}^{\mu} \frac{\left|\bar{H}^{2}\right|(\sigma x)}{\sigma} d \sigma\right) \\
& \leq C\left|\mu^{-2}-\lambda^{-2}\right| \int_{\lambda}^{\mu} \frac{|\bar{H}(\sigma x)|^{2}}{\sigma} d \sigma .
\end{aligned}
$$

Notice that, for $\mu \geq \lambda>1$,

$$
\int_{S^{n-1}} d x \int_{\lambda}^{\mu} \frac{|\bar{H}(\sigma x)|^{2}}{\sigma} d \sigma \leq \int_{0}^{\infty} \int_{S^{n-1}} \frac{|\bar{H}(\sigma x)|^{2}}{(1+\sigma)^{n}} \sigma^{n-1} d x d \sigma \leq C .
$$

The last inequality follows from the inequality $(\mathrm{K})$. Therefore, we have the estimate $(* *)$ :

$$
\int_{S^{n-1}}\left|f_{\lambda}(x)-f_{\mu}(x)\right|^{2} d x \leq C\left|\mu^{-2}-\lambda^{-2}\right|
$$

This implies that $\left(f_{\lambda}\right)$ is a Cauchy sequence in $L^{2}\left(S^{n-1}\right)$. Let $f_{\infty}$ be its unique limit. Since $\sup _{\mathbb{R}^{n}}\left|D f_{\lambda}\right|=\sup _{\mathbb{R}^{n}}|D f| \leq C_{0}$, the Arzela-Ascoli theorem tells us that $\left(f_{\lambda}\right)$ is compact in $C^{\alpha}\left(S^{n-1}\right), \forall \alpha \in(0,1)$. Therefore

$$
f_{\infty}(x)=\lim f_{\lambda}(x) \quad \text { uniformly on } S^{n-1},
$$

and

$$
f_{\infty}(r x)=r f_{\infty}(x), \quad \forall 0 \neq r \in \mathbb{R} .
$$

This finishes the proof of Theorem 1.1. 
In the following, we pose a question about the stability of self-similar solutions of (MCF). Let $f_{0}: \mathbb{R}^{n} \rightarrow \mathbb{R}^{k}$ be a smooth function with uniformly bounded (Lipschitz) gradient. Assume

$$
\lim _{\lambda \rightarrow \infty} f_{0 \lambda}=f_{0}^{\infty}, \quad \text { uniformly on } S^{n-1} .
$$

Assume $f: \mathbb{R}^{n} \times[0, \infty) \rightarrow \mathbb{R}^{k}$ such that $F(x, t)=(x, f(x, t))$ is a solution of (MCF) with the initial data $F(x, 0)=\left(x, f_{0}(x)\right)$. We ask if there is a smooth mapping $\hat{f}: \mathbb{R}^{n} \rightarrow \mathbb{R}^{k}$ such that $\hat{f}(\cdot, s) \rightarrow \hat{f}(\cdot)$ uniformly on compact subsets of $\mathbb{R}^{n}$ as $s \rightarrow \infty$. Here $\hat{f}$ is defined by

$$
\hat{f}(x, s)=t^{-\frac{1}{2}} f(\sqrt{t} x, t), s=\frac{1}{2} \log t, 0 \leq s<\infty \text { with } t \geq 1 .
$$

A related stability result is done by one of us in Ma 2003.

According to the remark of the referee, the codimension 1 case is settled in reference Stavrou 1998 with the trivial cone as only possible limit. A nice question now is that, can one give a condition that enforces the trivial cone in higher codimension? In Stavrou 1998, the stability for codimension 1 entire graphs with bounded gradient is treated - showing that they converge to asymptotically expanding solutions if they have a unique tangent cone at infinity. (This is of course not so relevant for the present paper, but may be related to our result in an interesting way).

\section{ACKNOWLEDGMENTS}

The work of Ma is partially supported by the key 973 project of China. We thank the referee for a useful suggestion.

\section{RESUMO}

Nesta nota, consideramos imersões auto-semelhantes do fluxo de curvatura média, e mostramos que uma solução em forma de gráfico da equação de soliton converge diferencialmente, contanto que tenha derivada limitada, para um gráfico cuja função tem propriedades especiais (V. Teorema 1.1).

Palavras-chave: Soliton, Auto-similar, Fluxo de curvatura média.

\section{REFERENCES}

EcKer K And Huisken G. 1989. Mean curvature evolution of entire graphs. Ann Math 130: 453-471.

Huisken G. 1989. Asymptotic behavior for singularities of the mean curvature flow. J Diff Geom 231: 285-299.

Huisken G. 1993. Local and global behavior of hypersurfaces moving by mean curvature flow. Proc. of Symposia in Pure Math 54 (Part I): 175-191.

MA L. 2003. B-sub-manifolds and their stability. math.DG/0304493.

SIMON L. 1983. Asymptotics for a class of nonlinear evolution equations, with applications to geometric problems, Ann Math 118: 525-571.

Stavrou N. 1998. Selfsimilar solutions to the mean curvature flow. J Reine Angew Math 499: 189-198. 